\title{
QUALITATIVE THEORY OF PARTIAL DIFFERENCE EQUATIONS (I): OSCILLATION OF NONLINEAR PARTIAL DIFFERENCE EQUATIONS
}

\author{
SUI SUN CHENG AND BING-GEN ZHANG
}

\begin{abstract}
This paper is concerned with a class of nonlinear partial difference equations with delay. By means of an averaging technique, and several oscillation criteria for ordinary recurrence relations, we are able to establish several oscillation criteria for the partial difference equations.
\end{abstract}

1. Functional parabolic equations of the form

$$
u_{t}=a(t) u_{x x}-p(t) u(x, t-\sigma),
$$

where $\sigma$ is the delay, have been studied by a number of authors (see for example [1, Chapter 6]). Such equations can be used to model problems of population dynamics with spatial migrations, chemical reactions, etc., where the dependent variable $u(x, t)$ changes in a continuous manner. However, in population dynamics where the population density varies in a seasonal manner and settlements are allowed only in concentrated locations, it is more appropriate to consider partial difference equations with delay.

In this paper, we consider a class of partial difference equations of the form

$$
\triangle_{2} u(i, j)=a(j) \triangle_{1}^{2} u(i-1, j)-q(i, j) f(u(i, j-\sigma)), \quad 1 \leq i \leq n, j \geq 0
$$

where the delay $\sigma$ is a nonnegative integer, $a(j)>0$ for $j \geq 0$ and $f$ is a real function defined on $R$. The real function $u(i, j)$ is dependent on integral variables $i, j$ which satisfy $0 \leq i \leq n+1$ and $j \geq-\sigma$. The partial difference $\Delta_{2} u(i, j)$ is defined by

$$
\triangle_{2} u(i, j)=u(i, j+1)-u(i, j)
$$

while

$$
\triangle_{1} u(i, j)=u(i+1, j)-u(i, j)
$$

Received January 12, 1994.

1991 Mathematics Subject Classification: 39A10, 35R10.

Key words and phrases: Nonlinear partial difference equation, oscillation. 
and the usual difference operator $\triangle$ is defined by

$$
\Delta v(k)=v(k+1)-v(k)
$$

As a consequence,

$$
\triangle_{1}^{2} u(i-1, j)=\triangle_{1}\left(\triangle_{1} u(i-1, j)=u(i+1, j)-2 u(i, j)+u(i-1, j) .\right.
$$

We shall assume that $u(i, j)$ is subject to the conditions

$$
\begin{gathered}
u(0, j)+\alpha(j) u(1, j)=0, \quad j \geq 0 \\
u(n+1, j)+\beta(j) u(n, j)=0, \quad j \geq 0
\end{gathered}
$$

and

$$
u(i, j)=\rho(i, j), \quad-\sigma \leq j \leq 0, \quad 0 \leq i \leq n+1,
$$

where $\alpha(j)+1 \geq 0$ and $\beta(j)+1 \geq 0$ for $j \geq 0$.

Given an arbitrary function $\rho(i, j)$ which is defined for $-\sigma \leq j \leq 0$ and $0 \leq i \leq n+1$, we can show that a solution to $(1-4)$ exists and is unique. Indeed, from $(1)$, we see that

$$
\begin{aligned}
& u(i, 1)=a(0) \rho(i+1,0)-(1-2 a(0)) \rho(i, 0)+a(0) \rho(i-1,0)-q(i, 0) f(\rho(i,-\sigma)), 1 \leq i \leq n, \\
& u(0,1)=-\alpha(1) u(1,1), \quad u(n+1)=-\beta(1) u(n, 1) .
\end{aligned}
$$

Inductively, we see that $\{u(i, j+1)\}_{i=1}^{n+1}$ is determined uniquely by $\{u(i, k)\}_{i=0}^{n+1}, k \leq j$.

For related studies of partial difference equations, see Cheng $[8,9,10]$.

2. Let $v(i, j)$ be a real function defined for $0 \leq i \leq n+1$ and $j \geq-\sigma$. Suppose there is some nonnegative integer $T$ such that $v(i, j)>0$ for $1 \leq i \leq n$ and $j \geq T$, then $v(i, j)$ is said to be eventually positive. An eventually negative $v(i, j)$ is similarly defined. The function $v(i, j)$ is said to be oscillatory for $1 \leq i \leq n$ and $j \geq 0$ if it is neither eventually positive nor eventually negative. Equation (1) is said to be oscillatory if every solution of $(1-4)$ is oscillatory.

To motivate our approach to equation (1), assume first that $q(i, j) \equiv q(j)$ for $1 \leq$ $i \leq n$ and $j \geq 0$, and that $f(x)=x$ for all $x$ in $R$. Then equation (1) takes the form

$$
\triangle_{2} u(i, j)=a(j) \triangle_{1}^{2} u(i-1, j)-q(j) u(i, j-\sigma), \quad 1 \leq i \leq n, j \geq 0 .
$$

If $(5)$ has an eventually positive solution $u(i, j)$ which is positive for $j \geq T$, then from (5), we have

$$
\sum_{i=1}^{n} \triangle_{2} u(i, j)=a(j) \sum_{i=1}^{n} \triangle_{1}^{2} u(i-1, j)-q(j) \sum_{i=1}^{n} u(i, j-\sigma),
$$


or,

$$
\Delta_{2} \sum_{i=1}^{n} u(i, j)=a(j)\{-(\beta(j)+1) u(n, j)-(\alpha(j)+1) u(1, j)\}-q(j) \sum_{i=1}^{n} u(i, j-\sigma)
$$

This implies

$$
\triangle v(j)+q(j) v(j-\sigma) \leq 0, \quad j \geq T+\sigma
$$

where

$$
v(j)=\sum_{i=1}^{n} u(i, j)
$$

As a consequence, we can obtain oscillation theorems for (5) provided that the relation (6) does not have an eventually positive solution. To this end, we recall a resu!t of Ladas et al. [2, Theorem 7.6.1] which states that if $\sigma$ is a positive integer, $q(n) \geq 0$ for $n \geq 0$ and

$$
\lim \inf _{n \rightarrow \infty}\left\{\frac{1}{\sigma} \sum_{j=n-\sigma}^{n-1} q(j)\right\}>\frac{\sigma^{\sigma}}{(\sigma+1)^{\sigma+1}}
$$

then (6) cannot have an eventually positive solution.

Another result is provided in Qian and Yan [3, Corollary 3] and Lalli and Zhang [4, Theorem 2.1] which states that if $q(j) \geq 0$ for $j \geq 0$, then the recurrence relation (6) has an eventually positive solution if and only if

$$
\Delta x(j)+q(j) x(j-\sigma)=0, \quad j \geq 0
$$

has an eventually positive solution. In view of this result, any oscillation theorem for (7) is also an oscillation theorem for (5).

In the results mentioned above, $q(n)$ is assumed to be nonnegative for $n \geq 0$. A recent paper by Yu et al. [5] considers oscillations of the equation (7) in which $q(j)$ may take on positive or negative values. Several of the oscillation criteria stated in this paper can be modified to yield oscillation criteria for the recurrence relation (6). Our interest, however, is in the oscillation of the nonlinear equation (1) which will be taken up in the following section.

3. We now assume that $q(i, j) \geq 0$ for $1 \leq i \leq n$ and $j \geq 0$. Let

$$
Q(j)=\min \{q(i, j) \mid 1 \leq i \leq n\} .
$$

Assume further that $f(x)$ is a nonnegative, nondecreasing and convex function on $(0, \infty)$.

If $u(i, j)$ is an eventually positive solution of (1) such that $u(i, j)>0$ for $1 \leq i \leq n$ and $j \geq T$. Then from (1), we have

$$
\frac{1}{n} \sum_{i=1}^{n} \Delta_{2} u(i, j)=\frac{a(j)}{n} \sum_{i=1}^{n} \Delta_{1}^{2} u(i-1, j)-\sum_{i=1}^{n} \frac{q(i, j)}{n} f(u(i, j-\sigma)) .
$$


Since $f$ is convex,

$$
\sum_{i=1}^{n} \frac{q(i, j)}{n} f(u(i, j-\sigma)) \geq Q(j) f\left[\frac{1}{n} \sum_{i=1}^{n} u(i, j-\sigma)\right] .
$$

Thus

$$
\triangle w(j)+Q(j) f(w(j-\sigma)) \leq 0, \quad j \geq 0
$$

has an eventually positive solution

$$
w(j)=\frac{1}{n} \sum_{i=1}^{n} u(i, j) .
$$

We have thus shown the following result.

Lemma 1. Suppose $\sigma$ is a nonnegative integer. Assume that $a(j) \geq 0, \sigma(j) \geq$ -1 and $\beta(j) \geq-1$ for $j \geq 0$, that $q(i, j) \geq 0$ for $1 \leq i \leq n$ and $j \geq 0$, and that $f(x)$ is a nonnegative, nondecreasing and convex function on $(0, \infty)$. If equation (1) has an eventually positive solution, so does equation (9), where $Q(j)$ is defined by $(8)$.

In order to obtain oscillation theorems for equation (1), we first establish a result which is analogous to that of Ladas et al. mentioned in the last section. The proof is also similar to that of their result [2, Theorem 7.5.1].

Lemma 2. Let $\sigma$ be a positive integer. Let $Q(j) \geq 0$ for $j \geq 0$ and $f(x) a$ positive nondecreasing and convex function on $(0, \infty)$ such that

$$
\lim _{x \rightarrow \infty} \frac{x}{f(x)}=M>0
$$

If relation (9) has an eventually positive solution, then the following inequality holds:

$$
\lim \inf _{n \rightarrow \infty}\left\{\frac{1}{\sigma} \sum_{j=n-\sigma}^{n-1} Q(j)\right\} \leq \frac{M \sigma^{\sigma}}{(\sigma+1)^{\sigma+1}}
$$

Proof. Let $\{v(i)\}$ be an eventually positive solution of (9) such that $v(i)>0$ for $j \geq T-\sigma$. Then from (9), $\Delta v(i)<0$ for $i \geq T-\sigma$ and

$$
v(i)+\sum_{j=T}^{i} Q(j) f(v(j-\sigma)) \leq v(T) .
$$

Thus $\lim _{i \rightarrow \infty} v(i)$ exists and

$$
\sum_{j=N}^{\infty} Q(j) f(v(j-\sigma))<\infty
$$


for all large $N$. Assume to the contrary that (10) does not hold. Then

$$
\sum_{i=N}^{\infty} Q(i)=\infty
$$

so that $v(i) \rightarrow 0$ as $i \rightarrow \infty$.

From (9), we have

$$
\triangle v(n)+Q(n) f(v(n)) \leq \Delta v(n)+Q(n) f(v(n-\sigma)) \leq 0,
$$

so that

$$
Q(n) \leq \frac{v(n)}{f(v(n))}\left\{1-\frac{v(n+1)}{v(n)}\right\}
$$

and

$$
\frac{1}{\sigma} \sum_{i=n-\sigma}^{n-1} Q(i) \leq \frac{1}{\sigma} \sum_{i=n-\sigma}^{n} \frac{v(i)}{f(v(i))}\left\{1-\frac{v(i+1)}{v(i)}\right\} .
$$

set $\rho=M \sigma^{\sigma} /(\sigma+1)^{\sigma+1}$. Choose a constant $\tau$ such that for sufficiently large $n$,

$$
\rho<\tau \leq \frac{1}{\sigma} \sum_{i=n-\sigma}^{n-1} Q(i) .
$$

Then since $v(i)$ converges to zero,

$$
\frac{v(n)}{f(v(n))} \leq M+\epsilon \text { for all large } n,
$$

where $0<\epsilon<((\tau / \rho)-1) M$, so that

$$
\tau \leq(M+\epsilon) \frac{1}{\sigma} \sum_{i=n-\sigma}^{n}\left\{1-\frac{v(i+1)}{v(i)}\right\} \leq(M+\epsilon)\left\{1-\left[\frac{v(n)}{v(n-\sigma)}\right]^{1 / \sigma}\right\}
$$

for all large $n$, where we have used the well known inequality between the arithmetic and geometric mean (see [2, Theorem 7.5.1]) in obtaining the second inequality. By means of the inequality [2, theorem 7.5.1],

$$
1-\lambda \leq\left\{\frac{\sigma^{\sigma}}{(\sigma+1)^{\sigma+1}}\right\}^{1 / \sigma} \lambda^{-1 / \sigma}, \quad 0<\lambda \leq 1
$$

we get

$$
\frac{v(n)}{v(n-\sigma)} \leq\left\{1-\frac{\tau}{M+\epsilon}\right\}^{\sigma} \leq \frac{\rho(M+\epsilon)}{\tau M}<1
$$


or

$$
\frac{v(n-\sigma)}{v(n)} \geq \frac{\tau M}{\rho(M+\epsilon)}>1
$$

which holds, say, for $n \geq n_{1}$.

If we apply the above inequality to (9), then instead of (11), we obtain

$$
\Delta v(n)+Q(n) f\left[\frac{\tau M}{\rho(M+\epsilon)} v(n)\right] \leq \Delta v(n)+Q(n) f(v(n-\sigma)) \leq 0 .
$$

A similar procedure then leads to

$$
\frac{v(n-\sigma)}{v(n)} \geq\left[\frac{\tau M}{\rho(M+\epsilon)}\right]^{2}, \text { for } n \geq n_{2}
$$

Inductively, we see that for every positive integer $k$, there is $n_{k}$ such that

$$
\frac{v(n-\sigma)}{v(n)} \geq\left[\frac{\tau M}{\rho(M+\epsilon)}\right]^{k}, \text { for } n \geq n_{k}
$$

We shall show that this is impossible under our assumption that (10) does not hold. Indeed, our assumption implies that there is a positive constant $c$ such that for all large $n$,

$$
\sum_{i=n-\sigma}^{n} Q(i)>c_{9} \sum_{i=n-\sigma}^{n^{\prime}} Q(i) \geq c / 2 \text { and } \sum_{i=n^{\prime}}^{n} Q(i) \geq c / 2, \text { where } n-\sigma \leq n^{\prime} \leq n .
$$

Since $v(i)$ is decreasing for large $i$, we have

$$
\begin{aligned}
v\left(n^{\prime}+1\right)-v(n-\sigma) & =-\sum_{i=n-\sigma}^{n^{\prime}} Q(i) f(v(i-\sigma)) \\
& \leq-f\left(v\left(n^{\prime}-\sigma\right)\right) \sum_{i=n-\sigma}^{n^{\prime}} Q(i) \leq \frac{-c}{2} f\left(v\left(n^{\prime}-\sigma\right)\right)
\end{aligned}
$$

and

$$
\begin{aligned}
v(n+1)-v\left(n^{\prime}\right) & =-\sum_{i=n}^{n} Q(i) f(v(i-\sigma) \\
& \leq-f(v(n-\sigma)) \sum_{i=n}^{n} Q(i) \leq \frac{-c}{2} f(v(n-\sigma)) .
\end{aligned}
$$

Combining (12) and (13), we obtain

$$
\begin{aligned}
0 & \geq v(n+1)-v\left(n^{\prime}\right)+\frac{c}{2} f(v(n-\sigma)) \frac{v(n-\sigma)}{v(n-\sigma)} \\
& \geq v(n+1)-v\left(n^{\prime}\right)+\frac{c}{2} \cdot \frac{f(v(n-\sigma))}{v(n-\sigma)}\left\{v\left(n^{\prime}+1\right)+\frac{c}{2} f\left(v\left(n^{\prime}-\sigma\right)\right)\right\}
\end{aligned}
$$


so that

$$
-v\left(n^{\prime}\right)+\left[\frac{c}{2}\right]^{2} \frac{f(v(n-\sigma))}{v(n-\sigma)} f\left(v\left(n^{\prime}-\sigma\right)\right) \leq 0
$$

and

$$
\frac{v\left(n^{\prime}-\sigma\right)}{v\left(n^{\prime}\right)} \leq\left[\frac{2}{c}\right]^{2} \frac{f(v(n-\sigma))}{v(n-\sigma)} \cdot \frac{v\left(n^{\prime}-\sigma\right)}{f\left(v\left(n^{\prime}-\sigma\right)\right)} \rightarrow\left[\frac{2}{c}\right]^{2} M^{2} .
$$

But this contradicts the fact that there is some positive integer $k$ such that

$$
\left[\frac{\tau M}{\rho(M+\epsilon)}\right]^{k}>\left[\frac{2}{c}\right]^{2} M^{2}
$$

The proof is complete.

At this point, we mention a result of the same nature which is essentially due to Erbe and Zhang [6] and its proof is only a slight modification of that of Theorem 2.5 in [6].

Lemma 3. Let $\sigma$ be a positive integer. Let $Q(j) \geq 0$ for $j \geq 0$ and $f(x)$ a positive, nondecreasing and convex function on $(0, \infty)$ such that

$$
\lim _{x \rightarrow \infty} \frac{x}{f(x)}=M>0
$$

If relation (9) has an eventually positive solution, then the following inequality holds:

$$
\limsup _{n \rightarrow \infty} \sum_{j=n-\sigma}^{n} Q(j) \leq M
$$

Next we establish a relation between the recurrence (9) and the following equation

$$
\triangle z(j)+Q(j) f(z(j-\sigma))=0, \quad j \geq 0 .
$$

For this purpose, we recall a fixed point theorem of Knaster and Tarski (see for example [2, Theorem 1.7.3.]).

Lemma 4. Let $B$ be a partially ordered Banach space with ordering $\leq$. Let $\Omega$ be a subset of $B$ such that the infimum of $\Omega$ belongs to $\Omega$ and every non-empty subset of $\Omega$ has a supremum which belongs to $\Omega$. Then every monotone mapping $T: \Omega \rightarrow \Omega$ has a fixed point in $\Omega$.

Lemma 5. Let $\sigma$ be a positive integer. Let $Q(i) \geq 0$ for $j \geq 0$ and

$$
\sum_{j=0}^{\sigma-1} Q(n+j)>0 \text { for all large } n
$$

Suppose further that $f(x)$ is a positive and nondecreasing function on $(0, \infty)$. 
If relation (9) has an eventually positive solution, so does equation (14).

Proof. Assume that $w(j)$ is an eventually positive positive solution of (9). Let $T$ be a nonnegative integer such that $T-2 \sigma \geq-\sigma$ and $w(j)>0$ for $j \geq T-2 \sigma$. Then from (9), $\Delta w(j)<0$ for $j \geq T-\sigma$ and

$$
w(i)+\sum_{j=T}^{i} Q(j) f(w(j-\sigma)) \leq w(T) .
$$

Thus $\lim _{j \rightarrow \infty} w(j)=w(\infty)$ exists (and is nonnegative),

$$
\sum_{j=T}^{\infty} Q(j) f(w(j-\sigma))<\infty
$$

and

$$
w(n) \geq w(\infty)+\sum_{j=n}^{\infty} Q(j) f(w(j-\sigma)), \quad n \geq T .
$$

Let $B$ denotes the partially ordered Banach space of all bounded real sequences $\{x(n)\}_{n=T}^{\infty}$ with the usual supremum norm and the componentwise defined partial ordering $\leq$. Let $\Omega$ be a subset of $B$ defined by

$$
\Omega=\{\{x(n)\} \in B \mid w(\infty) \leq x(n) \leq w(n), n \geq T\} .
$$

Note that inf $\Omega=\{w(n)\} \in \Omega$, and that for any nonempty subset $H=\left\{x^{\rho} \in B \mid \rho \in I\right\}$ of $\Omega$, the sequence $\left\{\sup _{\rho \in I} x^{\rho}(n)\right\}=\sup H$ also belongs to $\Omega$.

For every $x \in \Omega$, set $\widetilde{x}(n)=x(n)$ for $n \geq T$ and $\widetilde{x}(n)=x(T)+w(n)-w(T)$ for $T-\sigma \leq n<T$. Note that $0<\widetilde{x}(n) \leq w(n)$ for $T-\sigma \leq n<T$. Now define a mapping $S$ on $\Omega$ by

$$
(S x)(n)=w(\infty)+\sum_{j=n}^{\infty} Q(j) f(\widetilde{x}(j-\sigma)), \quad n \geq T .
$$

In view of (15), it is easily verified that the assumptions on $Q$ and $f$ imply that $S$ maps $\Omega$ into $\Omega$ and that $S$ is monotone. Thus all the hypotheses of Lemma 3 are satisfied. This implies that there is a sequence $z=\{z(n)\}$ in $\Omega$ such that $S z=z$. Clearly, $z$ satisfies equation (14) for all large $j$, and so the proof will be complete if we can show that $z(n) \neq 0$ for $n \geq T$. Indeed, as noted before, $z(n)>0$ for $T-\sigma \leq n<T$. Assume by induction that $z(n)>0$ for $T-\sigma \leq n<k$ where $T \leq k$, then

$$
\begin{aligned}
z(k) & =w(\infty)+\sum_{j=k}^{\infty} Q(j) f(\widetilde{z}(j-\sigma)) \geq w(\infty)+\sum_{j=k}^{k+\sigma-1} Q(j) f(\widetilde{z}(j-\sigma)) \\
& \geq w(\infty)+\min _{k \leq j \leq k+\sigma+1} f(\widetilde{z}(j-\sigma)) \sum_{j=k}^{k+\sigma-1} Q(j)>0 .
\end{aligned}
$$


The proof is complete.

4. Each of the Lemmas $1,2,3$ and 5 derived in the last section has a dual statement valid for eventually negative solutions. This is clear from the fact that $u(i, j)$ is a solution of $(1)$ if and only if $-u(i, j)$ is a solution of

$$
\triangle_{2} v(i, j)=a(j) \triangle_{1}^{2} v(i-1, j)-q(i, j) F(v(i, j-\sigma)), \quad 1 \leq i \leq n, j \geq 0
$$

where $F(t)=-f(-t)$ for all $t$; that $w(j)$ is a solution of $(9)$ if and only if $-w(j)$ is a solution of

$$
\Delta z(j)+Q(j) F(z(j-\sigma)) \geq 0, \quad j \geq 0
$$

etc.

As a consequence, these lemmas and their dual yield oscillation theorems. Several combinations are possible, but we shall confine ourselves to several examples.

Theorem 1. Let $\sigma$ be a positive integer. Let $Q(j)$ be defined by (8) and suppose it satisfies $Q(j) \geq 0$ for $j \geq 0$. Let $f(x)$ be a real function defined on $R$ such that $x f(x)>0$ for $x \neq 0, f(x)$ is nondecreasing on $R$ and $f(x)$ and $-f(-x)$ is convex on $(0, \infty)$ such that

$$
\lim _{x \rightarrow \infty} \frac{x}{f(x)}=M>0
$$

If

$$
\lim \inf _{n \rightarrow \infty}\left\{\frac{1}{\sigma} \sum_{j=n-\sigma}^{n-1} Q(j)\right\}>\frac{M \sigma^{\sigma}}{(\sigma+1)^{\sigma+1}}
$$

then equation (1) oscillates.

We remark that the above theorem is analogous to a result in [7, Theorem 3.1.2] which is concerned with ordinary nonlinear delay differential equation of the form

$$
y^{\prime}(t)+p(t) f(y(g(t))=0
$$

Theorem 2. Let $\sigma$ be a positive integer. Let $Q(j)$ be defined by (8) and suppose it satisfies $Q(j) \geq 0$ for $j \geq 0$. Let $f(x)$ be a real function defined on $R$ such that $x f(x)>0$ for $x \neq 0, f(x)$ is nondecreasing on $R$ and $f(x)$ and $-f(-x)$ is convex on $(0, \infty)$ such that

$$
\lim _{x \rightarrow \infty} \frac{x}{f(x)}=M>0
$$


If

$$
\limsup _{n \rightarrow \infty} \sum_{j=n-\sigma}^{n} Q(j)>M
$$

then equation (1) oscillates.

Theorem 3. Let $\sigma$ be a positive integer. Let $Q(j)$ be defined by (8) such that $Q(j) \geq 0$ for $j \geq 0$ and

$$
\sum_{j=0}^{\sigma-1} Q(n+j)>0 \text { for all large } n .
$$

Suppose further that $f(x)$ is a real function defined on $R$ such that $x f(x)>0$ for $x \neq 0, f(x)$ is nondecreasing on $R$ and $f(x)$ and $-f(-x)$ is convex on $(0, \infty)$. If every solution of (14) oscillates, then equation (1) oscillates.

\section{Acknowledgement}

The first author is partially supported by the NSC of R.O.C. The second author is partially supported by NNSF of China.

\section{References}

[1] D. D. Bainov and D. P. Mishev, "Oscillation Theory for Neutral Differential Equations with Delay," Adam Hilger, Bristol, 1991.

[2] I. Gyori and G. Ladas, Oscillation Theory of Delay Differrential Equations, Oxford Mathematical Monographs, Clarendon Press, Oxford, 1991.

[3] C. Qian and J. Yan, "Existence and comparison results of positive solutions of difference equations," Funkcialaj Ekvacioj, 35(1992), 581-595.

[4] B. S. Lalli and B. G. Zhang, "Oscillation and comparison theorems for certain neutral differrence equations," J. Austral. Math. Soc., Ser. B, 34(1992), 245-256.

[5] J. S. Yu, B. G. Zhang and X. Z. Qian, "Oscillations of delay difference equations with oscillating coefficients," J. Math. Anal. Appl., 177(1993), 432-444.

[6] L. H. Erbe and B. G. Zhang, "Oscillation of discrete analogues of delay equations," Differential and Integral Eq., 2(1989), 300-309.

[7] G. S. Ladde, V. Lakshmikantham and B. G. Zhang, Oscillation theory of differential equations with deviating arguments, Marcel Dekker, New York, 1987.

[8] S. S. Cheng and S. S. Lin, "Existence and uniqueness theorems for nonlinear difference boundary value problems," Utilitas Math., 39(1991), 167-186.

[9] S. S. Cheng and R.F. Lu, "Discrete Wirtinger's inequalities and conditions for partial differrence equations," Fasciculi Math., 23(1991), 9-24.

[10] S. S. Cheng, L. Y. Hsieh and Z. T. Chao, "Discrete Lyapunov inequality conditions for partial difference equations," Hokkaido Math. J., 19(1990), 229-239.

Department of Mathematics, Tsing Hua University, Hsinchu, Taiwan, 30043.

Department of Applied Mathematics, Ocean University of Qingdao, Qingdao, China. 